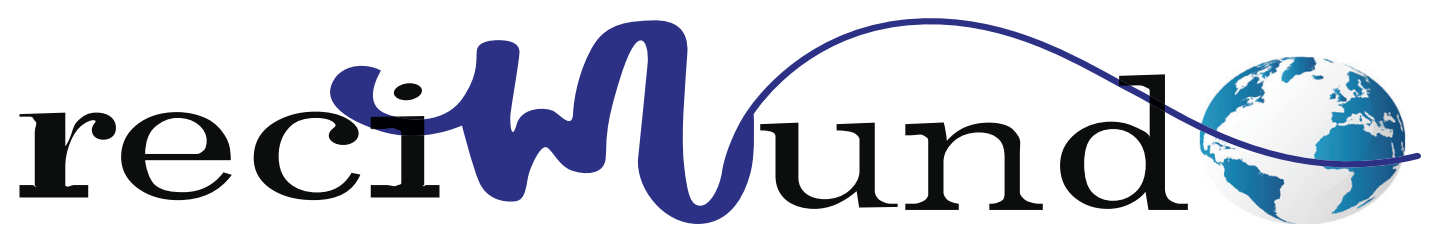

Revista Científica Mundo de la Investigación y el Conocimiento

DOI: 10.26820/recimundo/4.(4).noviembre.2020.392-402

URL: https://recimundo.com/index.php/es/article/view/1026

EDITORIAL: Saberes del Conocimiento

REVISTA: RECIMUNDO

ISSN: 2588-073X

TIPO DE INVESTIGACIÓN: Artículo de Investigación

CóDIGO UNESCO: Ciencias Médicas

PAGINAS: 392-402

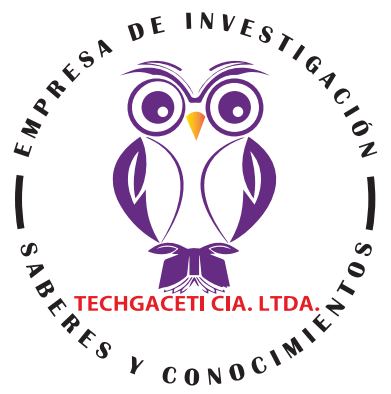

\title{
Eficacia del aceite de naranja en la desobturación de la gutapercha en los retratamientos de conductos
}

Efficacy of orange oil in the deobturation of gutta percha in root canal retreatments Eficácia do óleo de laranja na desobturação de guta-percha em retiros de canais radiculares

Juan Andrés Quinde Bayas ${ }^{1}$; María Teresa Noblecilla Soria²; Otto Vicente Campos Mancero ${ }^{3}$

RECIBIDO: 10/07/2020 ACEPTADO: 02/09/2020 PUBLICADO: 10/12/2020

1. Odontólogo de la Universidad de Guayaquil; Guayaquil, Ecuador; andres.quindeb@ug.edu.ec; (D) https://orcid.org/00000002-3142-889X

2. Magister en Ciencias Internacionales y Diplomacia; Especialista en Endodoncia; Doctora en Odontología; Universidad de Guayaquil, Ecuador; maria.noblecillas@ug.edu.ec; iD https://orcid.org/0000-0002-5432-7717

3. Magister en Investigación Clínica y Epidemiológica; Diploma Superior en preparación de Multirradiculares; Diploma Superior en Sistemas de Educación Superior Modular Basados en créditos Acumulables y Transferibles; Diploma Superior en Diseño Curricular por Competencias; Doctor en Odontología; Universidad de Guayaquil, Ecuador; otto.camposm@ug.edu. ec; (iD https://orcid.org/0000-0003-0656-3747

CORRESPONDENCIA

Juan Andrés Quinde Bayas

otto.camposm@ug.edu.ec

Guayaquil, Ecuador

(C) RECIMUNDO; Editorial Saberes del Conocimiento, 2020 


\section{RESUMEN}

La desobturación del conducto radicular, es un procedimiento cotidiano, por lo tanto, su ejecución debe ser realizada con instrumentos y materiales inocuos para evitar perjudicar los tejidos. El uso de disolventes de gutapercha tales como el aceite de naranja, es uno de los métodos utilizados para eliminar el contenido del conducto radicular. El objetivo de este trabajo fue establecer la eficacia que presentaba el aceite de naranja en la desobturación de los conductos radiculares. La metodología aplicada, fue de tipo cualitativa, experimental y descriptiva. Para este trabajo se utilizó aceite de esencia de naranja, jeringa de insulina de $1 \mathrm{ml}$, radiografías periapicales, 60 piezas dentarias unirradiculares y birradiculares, con tratamiento de conducto realizado y en los que se colocó seis gotas de aceite de esencia de naranja y se lo dejo actuar por 30 segundos, de los resultados obtenidos se pudo observar que el cálculo realizado en la desobturación de conducto, presentaba un coeficiente de correlación 0,96 y un tiempo promedio para la desobturación total del diente de 4 minutos con 45 segundo, además se concluyó que el aceite de naranja, es un disolvente natural muy efectivo como el xilol en la desobturación de la gutapercha.

Palabras clave: Desobturación, aceite de naranja, gutapercha, destilación, disolvente, endodoncia.

\section{ABSTRACT}

Root canal desobturation is a daily procedure, therefore its execution must be performed with harmless instruments and materials to avoid tissue damages. The use of gutta-percha solvents such as orange oil is one of the methods employed to eliminate the root canal content; it has proven to be effective for this purpose. The objective of this research is to establish the effectiveness of orange oil in the desobturation of root canals. The methodology applied was qualitative, experimental and descriptive. For this study, 60 single-rooted and double-rooted teeth were used with root canal treatment in which six drops of essential orange oil were placed. From the results obtained, it was observed that the calculation made in canal desobturation had a correlation coefficient 0.96 , thus obtaining very reliable data in the investigation. In all, orange oil is a very effective solvent in gutta-percha desobturation.

Keywords: Desobturation, orange oil, gutta-percha, distillation, solvent, endodontia.

\section{RESUMO}

A desobturação do canal radicular é um procedimento diário, portanto sua execução deve ser realizada com instrumentos e materiais inofensivos para evitar danos aos tecidos. O uso de solventes gutta-percha como o óleo de laranja é um dos métodos empregados para eliminar o conteúdo do canal radicular; provou ser eficaz para este fim. O objetivo desta pesquisa é estabelecer a eficácia do óleo de laranja na desobturação dos canais radiculares. A metodologia aplicada foi qualitativa, experimental e descritiva. Para este estudo, foram utilizados 60 dentes de raiz simples e dupla com tratamento de canais radiculares nos quais foram colocadas seis gotas de óleo essencial de laranjeira. A partir dos resultados obtidos, observou-se que o cálculo feito na desobturação do canal teve um coeficiente de correlação de 0,96, obtendo-se assim dados muito confiáveis na investigação. No total, o óleo de laranja é um solvente muito eficaz na desobturação de guta-percha.

Palavras-chave: Desobturação, óleo de laranja, gutta-percha, destilação, solvente, endodontia. 


\section{Introducción}

Los fracasos endodóntico, se pueden dar por parte del operador o por una anomalía que se encuentra dentro del conducto radicular, las causas principales para poder realizar un retratamiento endodóntico, es cuando se quedan bacterias dentro del conducto y para poder realizar la extracción del material obturado necesitamos de algún solvente químico, que nos facilite de manera más fácil, la remoción del material.

Al realizar la reintervención endodóntica, se presenta diversos tipos de complicaciones que van desde el endurecimiento considerable de la gutapercha, hasta la fractura del instrumento utilizado para la desobturación, además hay que tomar en cuenta el tipo de cemento endodóntico con el que se selló el conducto, puesto que según su composición, va a contribuir a su fácil o complicado retiro; todos estos factores nos obligan a aplicar diversas técnicas sean estas unitarias o híbridas (Magaly, 2014), para lograr el completo vaciado del conducto radicular y de esta manera, minimizar el contenido bacteriano para alcanzar un pronóstico favorable al diente.

El procedimiento de desobturación del conducto radicular, debe causar el mínimo nivel de agresividad e injuria hacia la estructura dentaria, por lo tanto la selección del método con el que se realizará la reintervención, debe contar con instrumentos y materiales apropiados para lograr la eliminación completa del contenido del conducto, así como también, el respeto a los tejidos.

En la literatura se mencionan algunas técnicas, tales como; la mecánica, térmica, química, utilizando tecnología, entre otros, cada una presenta debilidades y fortalezas, es así, que el operador debe tener entrenamiento para aplicarlas cuando el caso amerite; es decir, personalizar el método de desobturación para cada pieza dentaria.
Este trabajo, considera el método químico el cual, utilizando el aceite esencial de naranja en contacto con la gutapercha dentro del conducto radicular de piezas extraídas, reacciona con un efecto disolvente dentro de la superficie dentaria, permitiendo observar su comportamiento y poder medir el tiempo de remoción.

Entre las técnicas de desobturación de conductos encontramos la térmica, mecánica, química, ultrasónica y con limas rotatorias (Galiana, Gualdoni, Lugo, Montiel, \& Pelaez, 2018) (Hofmann, y otros, 2018); en este trabajo nos enfocaremos en el método quími$\mathrm{co}$, el cual usa solventes que reblandecen a la gutapercha y algunos hasta el cemento endodóntico facilitando la permeabilización de los conductos (Magaly, 2014), Entre los más conocidos tenemos al Eucaliptol, xilol, cloroformo, Endosolv E, Endosolv R, DMS IV (Dentsply/Maillefer) Aceite de trementina, aceite de naranja y aceite de limón.

El uso de aceites esenciales en endodoncia está creciendo, debido a su seguridad y biocompatibilidad (Hanan, 2016). El aceite de naranja, se obtiene de la cascara, semilla y pulpa, en estudios reciente el aceite se presentan propiedades antimicrobianas y se destaca por su baja citotoxicidad y buena capacidad para disolver el material de sellado del conducto radicular (Zuñiga, 2016), unas de las ventajas principales, es su nula toxicidad (Zuñiga, 2016), ya que son de consumo humano y su bajo costo, las desventajas del aceite de naranja, es su elaboración ya que el procedimiento es lento y demora horas, para poder obtener el producto y esto se lo aplicaría en la desobturación de la gutapercha.

Dentro de los disolventes naturales, tenemos el uso del aceite de naranja que cumplen las condiciones específicas convirtiéndolo en un disolvente principal en la remoción de los conductos radiculares (Ganchozo, 2015), este tipo de solvente natural, no presenta toxicidad y es de consumo humanos, 
ya que en el mercado se lo puede encontrar en bajo costo.

Cabe señalar que para poder obtener el aceite esencial de naranja, se procedió a investigar diferentes métodos para su obtención y aplicación en los conductos obturados para que actué de manera rápida, logrando disminuir el tiempo de trabajo para los especialistas en endodoncia, al mismo tiempo favorece la comodidad a los pacientes, ya que el aceite de naranja posee un aroma agradable (casado, 2018), otros beneficios por parte del operador es que es un producto de fácil elaboración y que en el mercado se lo puede obtener a bajo costo, el propósito principal es buscar la solución del problema al momento de la desobturación de los conductos radiculares.

El objetivo de este trabajo, es establecer la eficacia que presenta el aceite de naranja en la desobturación de conductos radiculares, así como también explicar los procedimientos para la obtención del aceite de naranja mediante el método de destilación y su exposición en función del tiempo al contactarse con el material obturador del conducto radicular.

\section{Metodología}

El método usado en el trabajo es inductivo-deductivo, porque permite explorar la documentación de lo general a lo particular, además analítico-sintético, debido a que permite resumir con criterio lo hallado en las fuentes primarias y secundarias de información en todo a lo que refiere los aceites esenciales y la desobturación de la gutapercha.

La técnica que se utilizó para la obtención del aceite esencial de naranja fue el método de la hidrodestilación con arrastre de vapor. Para poder realizar la investigación se seleccionaron 60 dientes obturados y se realizaron estudios radiográficos.

\section{Resultados}

En la investigación se lleva a cabo a través del método de la hidrodestilación con arrastre de vapor (casado, 2018), para lo cual se utiliza el equipo clevenger, un adaptador, un condensador y un termómetro, obteniendo un porcentaje de rendimiento óptimo, se procede a extraer el aceite esencial de naranja, considerando como insumo la cascara de la naranja que estará sumergida en agua destilada, Ilevándola a ebullición hasta alcanzar el punto de vapor, momento en el cual se extraer el aceite esencial.

Para poder obtener el aceite de naranja se emplea una relación, de por cada kilo de cascara de naranjas, se utilizan 2 litros de agua destilada; esto se somete a hidrodestilación con arrastre de vapor en una olla de presión; vertido todo en esta, se esperó a que llegue al punto de ebullición en un tiempo de (45 minutos aproximadamente), luego de quince minutos adicionales, el vapor comenzó a salir en emulsión (vapor-aceite esencial) hasta llegar al refrigerante donde, es condensado, cayendo en forma de gota, por un tiempo aproximado de 30 minutos desde la primera gota.

Este proceso de goteo, se recolecta en el Clevenger, las gotas que caen son una mezcla de aceite con agua, las cuales son separada en dos fase (agua-aceite esencial), al decantar el Clevenger, primero se retira el agua floral, hasta dejar el aceite esencial separado para ser utilizado en el experimento. Una vez obtenido el aceite esencial de naranja, se lleva a cabo la desobturación de los 60 dientes que estaban cementados con sealapex.

Al momento de su aplicación del aceite esencial de naranja en los dientes obturados, se colocó tres gotas en la entrada del conducto radicular y se lo dejo actuar por 30 segundos, esto para que comience hacer su efecto disolvente. Además, se hizo el ingreso al conducto con la lima hedström 45

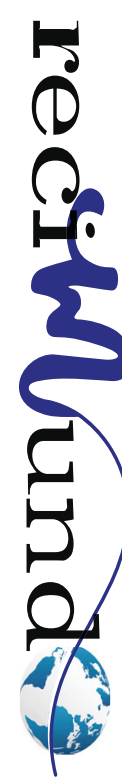


o 50 de la segunda serie, con movimientos de rotación hasta sentir una pequeña resistencia y comenzamos a retirar la lima para no ocasionar alguna fractura radicular.

Luego volvemos a ingresar con los mismos procedimientos anteriores pero de una manera más inmediata con movimientos de tracción, en la mitad del conducto radicular utilizamos 3 gotas que equivalen a 30 micras de aceite esencial, esto ayuda a reblandecer por completo la gutapercha. Finalmente se termina la desobturación cuando realizamos una toma radiográfica para asegurarnos que no se encuentre resto de material dentro del conducto.

\section{Experimento 1: obtención del aceite esencial de naranja}

Los procedimientos de investigación revelan detalles de este experimento, por tanto, se muestra evidencia de los procedimientos en el laboratorio, para la elaboración del aceite esencial con el método de hidrodestilación con vapor de arrastre.

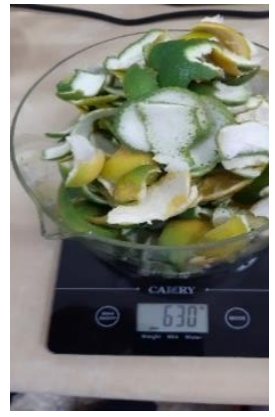

(a)

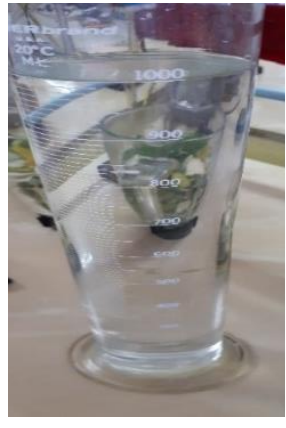

(b)

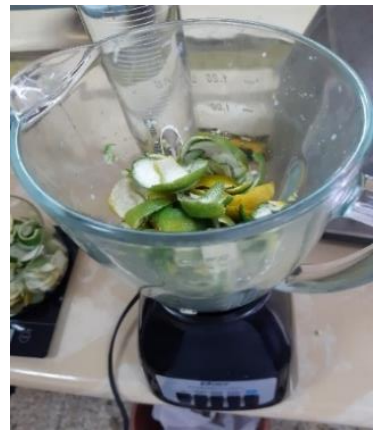

(c)

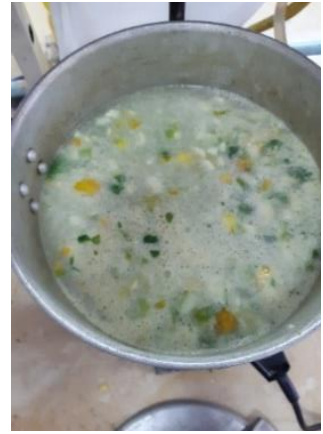

(d)

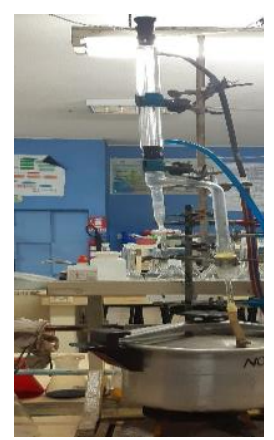

(e)

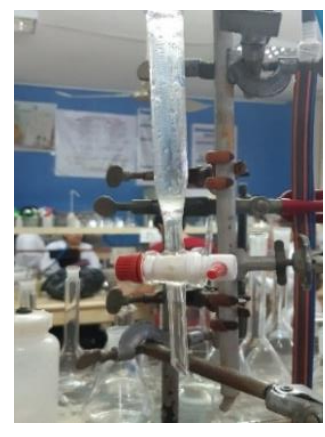

(f)

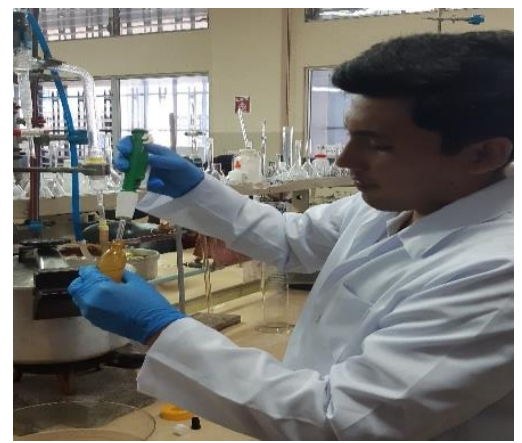

(g)

Imagen 1. Proceso de obtención del aceite esencial de naranja

Fuente: Los autores

Detalle: (a) Peso de la cascara de naranja 1Klgr; (b) Volumen del agua destilada 200ml; (c) Licuadora con los ingredientes; (d) Olla de presión c-on los ingredientes; (e) Sistema de la hidrodestilación con arrastre de vapor; (f) Clevenger para el proceso de goteo; (g) Obtención del aceite esencial de naranja. 


\section{Experimento 2: pruebas de desobturación en conos de gutapercha}

Se procede a mostrar el tiempo que tarda en remover la gutapercha con la incidencia del aceite esencial de naranja.

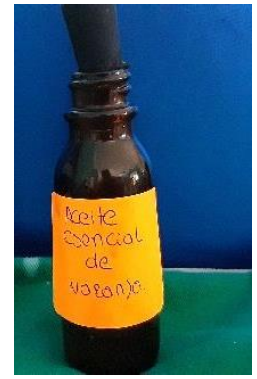

(a)

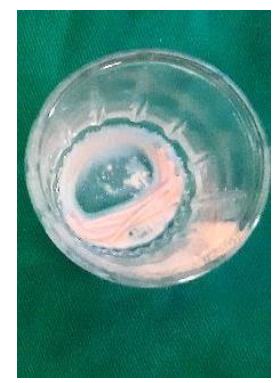

(b)

Aceite esencial de naranja Cono de gutapercha

Imagen 2. Desobturación en conos de gutapercha

Fuente: Los autores

Tiempo del aceite esencial de naranja: 2:40 debido a que el experimento no está con cemento Sealapex.

Experimento 3: Procedimiento para realizar la desobturación de los conos de gutapercha

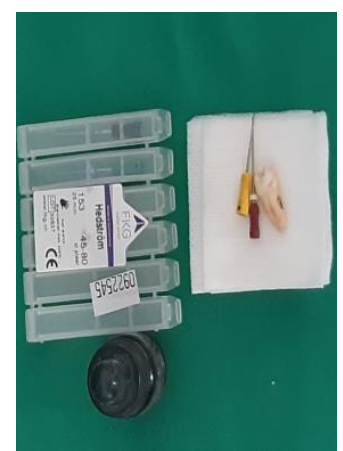

(a)

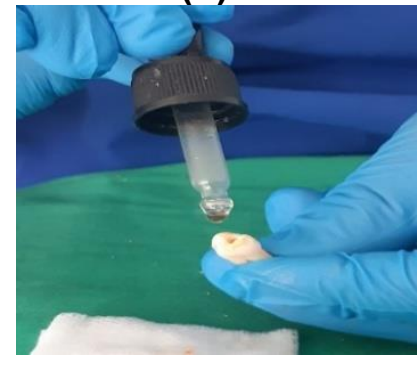

(e)

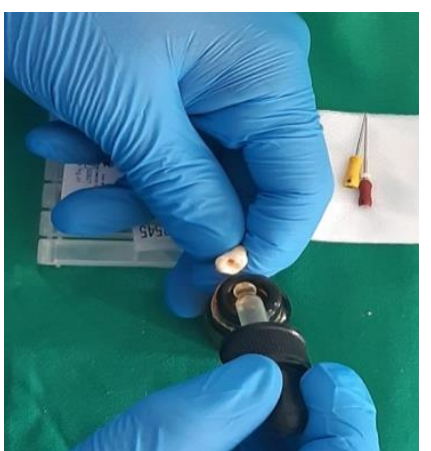

(b)

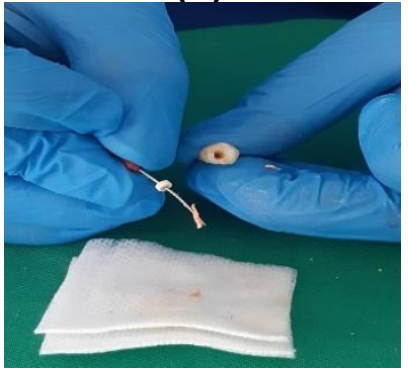

(f)

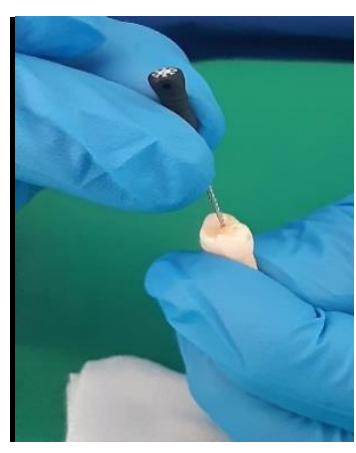

(c)

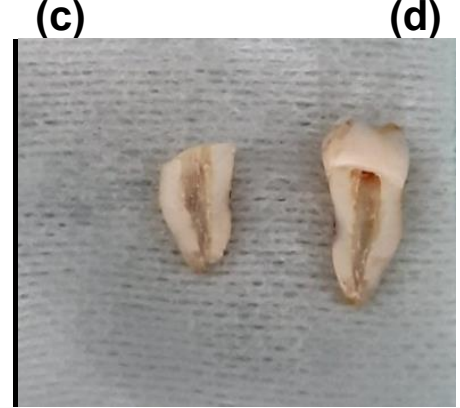

(g)

(d)
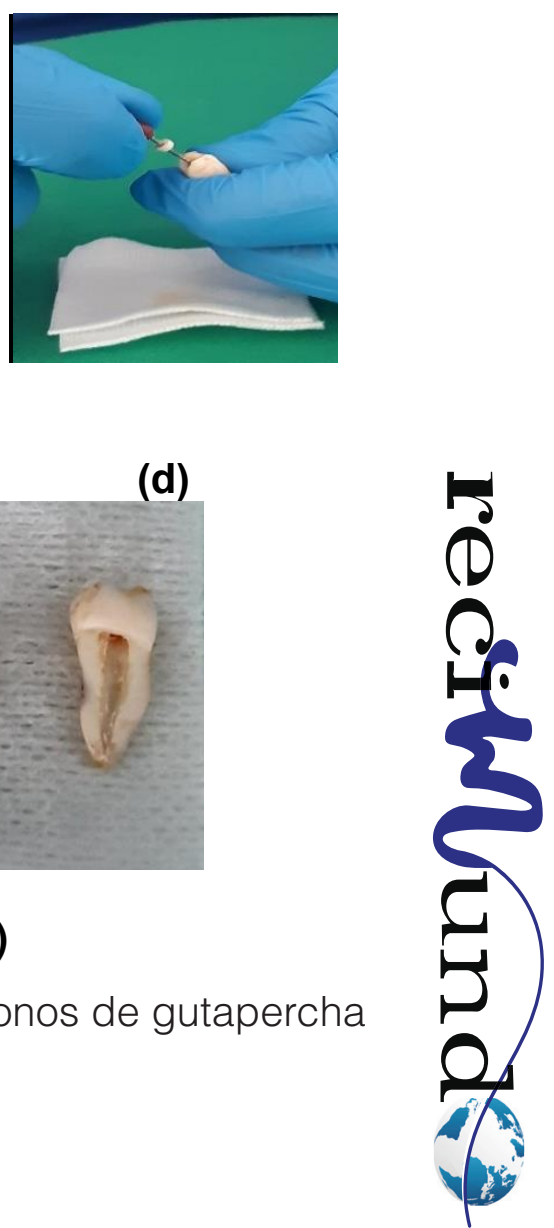
Detalle: (a) Limas herström; (b) Aplicación de las tres gotas de aceite esencial de naranja en la entrada del conducto; (c) Manipulación la pieza dentaria con movimientos de rotación; (d) Movimientos de tracción con la lima herström; (e) Colación de las tres gotas del aceite esencial en la mitad del conducto; (f) Desobturación total de la gutapercha; (g) Disección de la pieza dentaria.

En el ensayo obtuvimos $15 \mathrm{ml}$ de aceite esencial envasado en un frasco de vidrio hermético para que no se volatilice el aceite obtenido.

Tabla 1. Características del aceite esencial envasado

\begin{tabular}{lcc}
\hline Características del envase & Cantidad & Medida \\
\hline Capacidad del frasco & 41,77 & $\mathrm{gr}$ \\
Peso del frasco más el Aceite & 54,28 & $\mathrm{gr}$ \\
Medida del Aceite & 12,52 & $\mathrm{gr}$ \\
Volumen de Aceite & 15,00 & $\mathrm{ml}$ \\
\hline
\end{tabular}

Fuente: Los autores
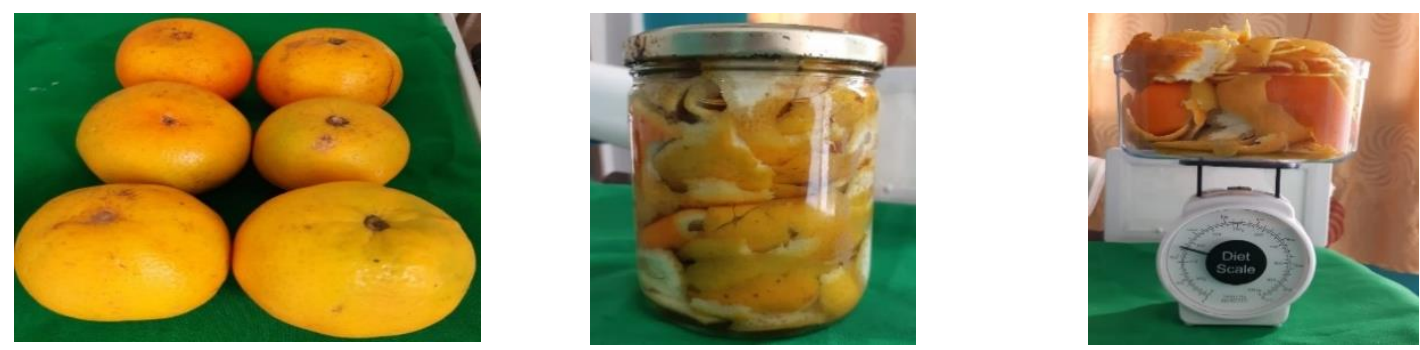

Imagen 4. Elaboración del aceite de naranja con la mescla de la cascara de naranja

Fuente: Los autores

Tabla 2. Datos experimentales del tiempo de reacción del solvente en los conductos

\begin{tabular}{|c|r|r|r|r|r|r|r|}
\hline MUESTRA & TIEMPO (seg) & MUETRA & TIEMPO (seg) & MUESTRA & TIEMPO (seg) & MUESTRA & TIEMPO (seg) \\
\hline 1 & 1,42 & 16 & 3,49 & 31 & 4,30 & 46 & 5,53 \\
\hline 2 & 2,00 & 17 & 3,50 & 32 & 4,35 & 47 & 6,00 \\
\hline 3 & 2,10 & 18 & 3,54 & 33 & 4,36 & 48 & 6,00 \\
\hline 4 & 2,11 & 19 & 3,55 & 34 & 4,37 & 49 & 6,30 \\
\hline 5 & 2,13 & 20 & 4,00 & 35 & 4,40 & 50 & 6,80 \\
\hline 6 & 2,19 & 21 & 4,00 & 36 & 4,40 & 51 & 6,90 \\
\hline 7 & 2,31 & 22 & 4,00 & 37 & 4,46 & 52 & 6,24 \\
\hline 8 & 2,47 & 23 & 4,10 & 38 & 5,00 & 53 & 6,20 \\
\hline 9 & 3,00 & 24 & 4,11 & 39 & 5,12 & 54 & 6,30 \\
\hline 10 & 3,13 & 25 & 4,12 & 40 & 5,12 & 55 & 6,30 \\
\hline 11 & 3,37 & 26 & 4,15 & 41 & 5,30 & 56 & 6,35 \\
\hline 12 & 3,40 & 27 & 4,20 & 42 & 5,30 & 57 & 6,46 \\
\hline 13 & 3,45 & 28 & 4,25 & 43 & 5,30 & 58 & 7,20 \\
\hline 14 & 3,45 & 29 & 4,30 & 44 & 5,37 & 59 & 7,40 \\
\hline 15 & 3,47 & 30 & 4,30 & 45 & 5,42 & 60 & 8,25 \\
\hline
\end{tabular}

Fuente: Los autores 
Tabla 3. Datos de la estadística descriptiva del tiempo del solvente

\begin{tabular}{lr}
\hline \multicolumn{2}{c}{ Tiempo (Segundos) } \\
\hline \\
Media & 285,067797 \\
Error típico & 11,8996109 \\
Mediana & 275 \\
Moda & 240 \\
Desviación estándar & 91,4026455 \\
Varianza de la & \\
muestra & 8354,4436 \\
& - \\
Curtosis & 0,38617358 \\
Coeficiente de & \\
asimetría & 0,22756078 \\
Rango & 385 \\
Mínimo & 120 \\
Máximo & 505 \\
Suma & 16819 \\
Cuenta & 59 \\
Nivel de confianza & \\
(95,0\%) & 23,8196591 \\
\hline
\end{tabular}

Fuente: Los autores

Tabla 4. Recolección de los datos experimentales

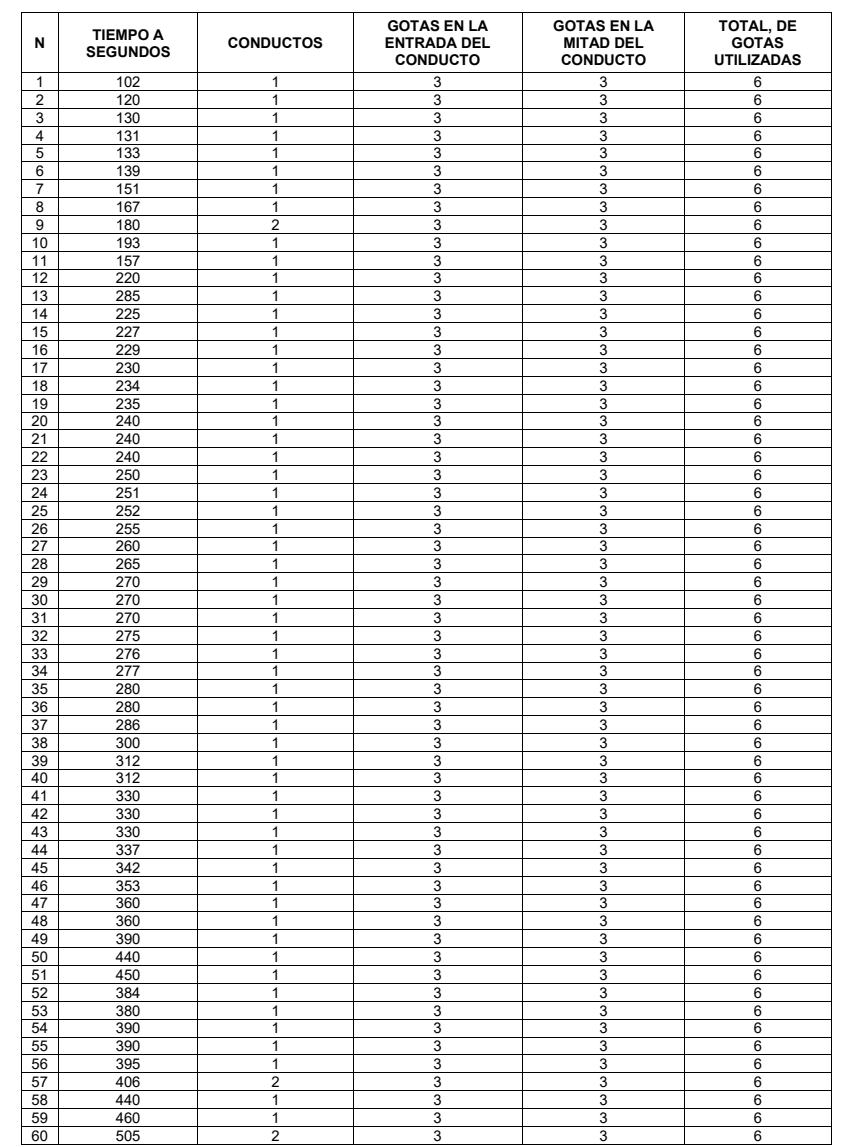


Para lograr el análisis de los retratamientos endodónticos sobre dientes extraídos, se obtuvieron resultados positivo como se muestra en la tabla de recolección de datos experimentales, evidenciando una correlación de R2 de 0,9646 y un tiempo promedio para la remoción total de los dientes obturados con el aceite esencial de naranja de 4 minutos con 45 segundos como lo muestra la estadística descriptiva para ser desobturado, lo importante de la investigación, es que el aceite esencial de naranja en un solvente natural que no va ocasionar ningún tipo de problema al paciente al momento de su aplicación, ya que no es tóxico en comparación al xilol, que es un solvente químico, el aceite de naranja presenta un aroma agradable para la comodidad del paciente durante su retratamiento endodóntico, sin embargo, hay que tener en cuenta que los solventes químicos que ocasionan toxicidad ya no se encuentran en el mercado y el aceite de naranja cumple la función específica de ser un solvente eficaz en la desobturación de la gutapercha.

Los datos que al ser comparados por Sanchez \& Vedia (2016) en su trabajo "Análisis in vitro de la eficacia de dos solventes: aceite de naranja y eucaliptol empleados en la desobturación de conductos radiculares en la clínica odontológica univalle-cochabamba" quienes concluyeron que se logro demostrar que el Aceite de Naranja resulta más efectivo que el Eucaliptol en la remoción de los conos de gutapercha para la desobturación de los conductos radiculares en menor tiempo, permitiendo la penetración de $3 \mathrm{~mm}$ de una lima tipo $\mathrm{K}$, con estos resultados se afirma que el aceite esencial de naranja actua de una manera eficaz en los tratamientos de conductos.

\section{Conclusiones}

Al finalizar este trabajo, queda demostrado que la consulta a fuentes primarias y secundarias de información, permitieron construir los fundamentos teóricos con los cuales se logró realizar los experimentos.

La aplicación de la técnica de la hidrodestilación, permitió obtener el aceite esencial de naranja, con el cual se realizaron los experimentos para la desobturación de los conductos radiculares, encontrando resultados afines a otros trabajos, además este compuesto no presenta toxicidad y resulta de bajo costo para su uso.

El experimento 3, revela los tiempos que tarda el disolvente en resblandecer el material obturado, actuando sobre una base de 4 minutos con 45 segundos, además se muestra a través de la aplicación de la estadística descriptiva y su coeficiente de correlación de 0,96 que brinda fiabilidad al trabajo realizado.

Lo anterior permite comprobar la hipótesis planteada en este trabajo ya que el aceite esencial de naranja demuestra ser un disolvente efectivo en la desobturación de la gutapercha para los tratamientos de conducto. Considerando la efectividad de los cítricos, se sugiere emplear aceite esencial de limón en los retratamientos endodónticos, y establecer comparativos en su nivel de eficiencia.

Además para disminuir los tiempos de reacción del solvente se recomienda aumentar la cantidad de gotas en los experimentos.

Se recomienda utilizar el aceite esencial de naranja porque no presenta toxicidad y carece de un bajo índice de irritabilidad.

Se recomienda que la instrumentación se encuentre en óptimas condiciones para la correcta utilización del aceite esencial de naranja y lograr resultados de manera más rápida y eficiente.

Es recomendable el uso del aceite esencial de naranja, porque deja un aroma agradable y no queda grasoso al momento de ser empleado. 


\section{Bibliografía}

Aguilar, w., \& Mayid, B. (2010). retratamientos endodonticos, Reporte de dos casos clinicos. Idental, 4.

Andrea, F., \& Pastenes, A. (2018). Técnicas y sistemas actuales de obturación en endodoncia. revisión crítica de la literatura. KIRU., 87.

Begazo, J. (2017). "Estudio In Vitro para determinar la efectividad del óleo de naranja y el eucaliptol como disolvente de gutapercha en retratamientos endodónticos, de acuerdo al tiempo y peso. Universidad privada de tacna, 41.

casado, I. (2018). optimizacion de la extraccion de aceite esenciales por destilacion en corriente de vapor. Trabajo fin de grado para la obtencion del titulo de graduado en ingenieria en tecnologias indutriales, Universidad Politecnica de Madrid, Escuela tecnica superior de ingenieros industriales , Madrid.

Ceron, I., \& Cardona, C. (2011). Evaluaci'on del proceso integral para la obtencion de aceite esencial y pectina a partir de cascara de naranja. Scielo, 67,68

Galiana, M., Gualdoni, G., Lugo, C., Montiel, N., \& Pelaez, A. (2018). Tecnica manuales y mecanizadaen el tratamiento endodontico. San Gregorio, 9.

Ganchozo, E. (2015). Eficacia de diferentes atrayentes alimenticio"en cultivo de naranaja. Proyecto de Investigación previo a la obtencion del titulo de ingeniero, Universidad Tecnica Estatal de Quevedo, Facultad de ciencia agrarias, Quevedo.

Garcia, J. (2019). Accion del aceite esencial de limon en la reintervencion endodontica. Trabajo de grado previo a la obtencion del titulo de Odontologia, Universidad de Guayaquil, Facultad de Odontologia, Guayaquil .

Goldberg, F., \& Cantarini, C. (2014). El retratamiento endodontico. Divulgacion endodontica, 76.

Hanan, A. (2016). Disolución de un sellador agregado de trióxido mineral en solventes endodónticos en comparación con selladores convencionales. SciELO, 5.

Herrera, G. (2018). Análisis comparativo del xilol y eucaliptol en la desobturación de conductos radiculares. Estudio in vitro. Universidad de Guayaquil Facultad Piloto de Odontologia, 31.

Hidalgo, I. (2015). Estudio in-vitro retratamiento en dientes unirradiculares obturados con gutapercha; para observar la acción del solvente y su efecto sobre las paredes dentinarias; en compa- ración con técnica de desobturación mecánica. trabajo de titulacion, Universidad Internacional del Ecuador, Facultad de ciencia medicas de la salud y de la vidad escuela de odontologia, Guayaquil .

Hofmann, M., Olivares, S., Cadena, M., López, A., Rodríguez, E., \& Carrillo, A. (2018). Comparación de la Eficacia del extracto de naranja, D-limoneno y xilol en retratamientos de dientes obturados con gutapercha. Dentista y Paciente, 1.

Logroño, C. (2017). Comparacion de las tecnicas de obturacion empleadas en endodoncia ,tecnica cono unico, condensacion lateral, termoplastificada y tecnica con portador thermafil con nucleo de gutapercha y plastica y medir su eficacia mediante la revision bibliografica. colegio de ciencia de la salud, 30.

Lucarelli, A., \& Lucarelli, J. (2014). comparacion de la eficacia de la desobturacion total radicular utilizando una tecnica rotatoria niti con la tecnica manual estudio in vitro. Finis Terrae, 8.

Magaly, A. (2014). Técnicas químico mecánicas con la utilización de xilol en un. tesis para la obtencion del titulo de grado, Universidad Guayaquil, Facultad Piloto d Odontologia, Guayaquil.

Mariel Beatriz Galiana,Graciela Mónica Gualdoni,Carlos Lugo de Langhe,Natalia Belén Montiel,Alina Pelaez. (2018). Revisión de desobturación de gutapercha con limas manuales, Xilol y Reciproc. Scielo, 1.

Mejía, M. E. (2011). Evaluación in vitro de tres. ODONTOLOGÍA SANMARQUINA, 3.

Ramirez, M. (2017). comparacion in vitro de aceite esencial de las cascara de limon y el aceite esencial de cascara de naranja como solventes de gutapercha de uso endodontico. Tesis para obtener el grado academico de maestria en estomatologia, Universidad Nacional de Trujillo, Escuela de Posgrado en Ciencia Medicas, Trujillo.

Ramirez, M. I. (2017). Comparacion in vitritio del aceite de Limon y Nranja . Ramirez Monica Lucia , 6.

Ramírez, T. (2015). "Estudio comparativo in vitro del grado de limpieza de los conductos radiculares en los tercios medio y apical utilizando dos técnicas diferentes de desobturación en dientes unirradiculares obturados con la técnica de condensación lateral convencional". Tesis de Grado presentada como requisito previo para la obtención del título de Especialista en Endodoncia, Universidad Central del Ecuador, Facultad de Odontologia Instituto Superior de Investigacion y Posgrado, Quito.

Rosa, Z. C. (2014). Conservacion de zumo de naranja. Tesis de grado para la obtencion del titulo de 
ingenieria Agroindustrial, Universidad Laica Eloy Alfaro de Manabi, Facultad de ciencia Agropecuarias Especialidad Agroindustria, Manabi.

Sanchez, P., \& Vedia, C. (2016). Análisis in vitro de la eficacia de dos solventes: aceite de naranja y eucaliptol y eucaliptol empleados en la desobturación de conductos radiculares en la clínica odontológica univalle-cochabamba, gestión 2014. Revista Boliviana, 1.

Ulloa, S. (2017). Capacidad disolutiva del Eucaliptol y Aceite de Naranja frente a cementos Endodónticos (estudio in vitro) en la Universidad Católica de. Trabajo de titulacion previo a la obtencion del titulo odontologico, Universidad Catolica de Cuenca, Unidad academica de salud y bienestar, Cuenca.
Yances, S. ( 2018). Importancia de la producción de naranja en Caluma y el impacto que tiene en los festivales del cantón. Trabajo de titulación presentado como requisito para la obtención del título de Licenciado en Gastronomía, Universidad San Francisco de Quito, Colegio de Hospitalidad, Arte Culinario y Turismo, Quito.

Zuñiga, G. (2016). Estudio compar cativo in vitrio de tecnicas de desobturacion de conductos radiculares con solventes ,xilol versus aceite de naranja y su incidencia en la eliminacion de gutapercha. proyecto de ivestigacion previo a la obencion de titulo de odontologia, Universidad Regional Autonoma de los Andes, facultad de ciencias medicas carrera de odontologia, Ambato.

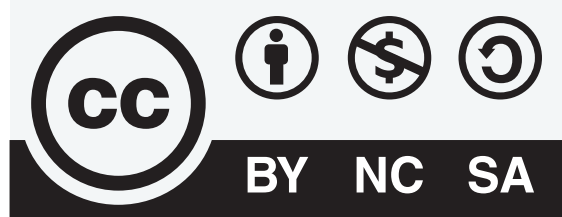 \\ CREATIVE COMMONS RECONOCIMIENTO-NOCO- MERCIAL-COMPARTIRIGUAL 4.0.}

\section{CITAR ESTE ARTICULO:}

Quinde Bayas, J. A., Noblecilla Soria, M. T., \& Campos Mancero, O. V. (2020). Eficacia del aceite de naranja en la desobturación de la gutapercha en los retratamientos de conductos. RECIMUNDO, 4(4), 392-402. https://doi.org/10.26820/ recimundo/4.(4).noviembre.2020.392-402 